\title{
The spectroscopic signature of roAp stars ${ }^{\star}$
}

\author{
T. Ryabchikova ${ }^{1,2}$, N. Nesvacil ${ }^{1,3}$, W. W. Weiss ${ }^{1}$, O. Kochukhov ${ }^{1,4}$, and Ch. Stütz ${ }^{1}$ \\ 1 Department of Astronomy, University of Vienna, Türkenschanzstrasse 17, 1180 Vienna, Austria \\ e-mail: first_name@astro.univie.ac.at \\ 2 Institute of Astronomy, Russian Academy of Sciences, Pyatnitskaya 48, 109017 Moscow, Russia \\ e-mail: ryabchik@inasan.rssi.ru \\ 3 European Southern Observatory, Casilla 19001, Santiago 19, Chile \\ ${ }^{4}$ Uppsala Astronomical Observatory, Box 515, 75120 Uppsala, Sweden
}

Received 2 April 2004 / Accepted 10 May 2004

\begin{abstract}
To reliably determine the spectroscopic signature of rapidly oscillating chemically peculiar (roAp) stars it is also necessary to investigate a sample of non pulsating chemically peculiar (noAp) as well as presumably "normal" stars. We describe in this study the sample of spectroscopically investigated stars and comment on the techniques used for the analysis. In particular we discuss ionization disequilibria of rare earths in roAp stars that distinguish them from noAp stars. In the light of the recently discovered pulsation of $\beta \mathrm{CrB}$ we see arguments that all magnetic $\mathrm{CP} 2$ stars up to a transition temperature of about $8100 \mathrm{~K}$ may be pulsating.
\end{abstract}

Key words. stars: abundances - stars: atmospheres - stars: chemically peculiar - stars: oscillations

\section{Introduction}

The detection of rapid oscillation in HD 101065 by D. Kurtz (1978) marked the discovery of a new class of pulsating stars, the roAp stars, and provided an important impetus to asteroseismology. Contrary to the other pulsating variables known at that time, roAp stars are a subgroup of already quite "abnormal" stars, the well-known but still not sufficiently understood chemically peculiar A-type stars. Their peculiar atmosphere causes considerable problems when determining stellar fundamental parameters, like $T_{\text {eff }}, \log g$ or metallicity with classical techniques calibrated on "normal" stars. As we have been developing the tools for a better understanding of CP star atmospheres by replacing various approximations by more physical descriptions, we decided to investigate also roAp stars spectroscopically and to determine their fundamental parameters, in particular their abundance spectrum relative to the sun and to non-pulsating Ap (noAp) stars with similar $T_{\text {eff }}$ and $\log g$.

In 1996 the only roAp star with a well investigated atmosphere (Kupka et al. 1996 - Paper I) was $\alpha$ Cir. Thanks to new observations and the availability of already archived spectra through collaborations with various institutions, the situation has changed significantly as has been illustrated in a progress report by Weiss et al. (2000).

* Based on observations obtained at the European Southern Observatory (La Silla, Chile), the Canadian-French-Hawaii telescope, the South Africa Astronomical Observatory, The Crimean Astrophysical Observatory and on numerous SIMBAD interrogations.
Atmospheric characteristics and abundances of at least the most important group of elements for 12 out of 32 known roAp stars are now available, including such a pathological case as Przybylski's star - HD 101065 (Cowley et al. 2000). Ryabchikova et al. (2001) analysed 6 roAp and 6 nonoscillating Ap (noAp) stars of similar effective temperatures and found a remarkable anomaly in Pr II - Pr III and Nd II Nd III line intensities in spectra of all investigated roAp stars. This anomaly was a difference of about 2 dex in the abundances derived from the first and second ions. In four noAp stars the same anomaly seemed to be marginally - if at all - present. In two noAp stars, HD 62140 and HD 115708, this REE anomaly was found and these stars were proposed as candidates for pulsation. Since that investigation, the same REE anomaly was found in another three roAp stars: HD 101065 (Cowley et al. 2000), HD 122970 (Ryabchikova et al. 2000), and HD 213637 (Kochukhov 2003). In order to put our conclusions about abundance characteristics of pulsating Ap stars on a solid statistical basis, it was necessary to increase the sample of nonpulsating Ap and A stars with a detailed abundance analysis.

\section{Program stars and observations}

The observing log of our program stars plus name, range in $\lambda$ and resolution of the spectrograph is given in Table 1. The spectra of most of the stars discussed in this paper were obtained at the European Southern Observatory (ESO), the South African Astronomical Observatory (SAAO), and the CanadaFrance-Hawaii Telescope (CFHT). Spectra of three stars were 
Table 1. Log of new spectroscopic observations. Type: "normal" non-pulsating Ap stars (Ap), pulsating Ap stars (roAp); S/N: signal-to-noise ratio of a typical pixel in the continuum; Obs.: observatory (CFHT: Canadian French Hawaii Telescope, ESO: European Southern Observatory, SAAO: South African Astronomical Observatory) and observer (FK: Kupka, KZ: Zwintz, OK: Kochukhov, PM: Mittermayer, WW: Weiss); $\Delta \lambda$ : wavelength range of the spectrum; $R$ : spectral resolution; $\langle\mathrm{H}\rangle$ : mean magnetic field modulus in Gauss.

\begin{tabular}{|c|c|c|c|c|c|c|c|}
\hline HD & Type & HJD & $S / N$ & Obs. & $\Delta \lambda(\AA)$ & $R$ & $\langle\mathrm{H}\rangle(\mathrm{G})$ \\
\hline 12098 & roAp & 52184.931 & 150 & CFHT: FK, WW & 6106-9188 & 115000 & $\approx 6500$ \\
\hline \multirow[t]{4}{*}{18610} & Ap & 51417.574 & 63 & SAAO: PM, KZ & $4400-7000$ & 37000 & 5700 \\
\hline & & 51417.599 & 68 & & & & \\
\hline & & 51417.620 & 66 & & & & \\
\hline & & 51417.642 & 63 & & & & \\
\hline \multirow[t]{2}{*}{29578} & Ap & 51600.518 & 125 & ESO: archive & $6127-6167$ & 122500 & 5134 \\
\hline & & 51946.538 & 225 & ESO: OK & $6126-6156$ & 123000 & 5537 \\
\hline 60435 & roAp & 51947.533 & 225 & ESO: OK & $6126-6156$ & 123000 & \\
\hline \multirow[t]{3}{*}{75445} & Ap & 51600.623 & 125 & ESO: archive & $6127-6167$ & 122500 & 2915 \\
\hline & & 51945.543 & 225 & ESO: OK & $6126-6156$ & 123000 & 2957 \\
\hline & & 51955.512 & 225 & & & & 2873 \\
\hline \multirow[t]{3}{*}{116114} & Ap & 51416.217 & 88 & SAAO: PM, KZ & $4400-7000$ & 37000 & 6200 \\
\hline & & 51416.232 & 89 & & & & \\
\hline & & 51600.699 & 125 & ESO: archive & $6127-6167$ & 122500 & 6047 \\
\hline 137909 & Ap & 52008.970 & 220 & CFHT: FK, WW & 6102-6192 & 115000 & 5432 \\
\hline$(\beta \mathrm{CrB})$ & & 52008.970 & 220 & & $5492-5572$ & 60000 & 5432 \\
\hline 137949 & roAp & 51416.270 & 101 & SAAO: PM, KZ & $4400-7000$ & 37000 & 5000 \\
\hline \multirow[t]{3}{*}{ (33 Lib) } & & 51416.284 & 114 & & & & \\
\hline & & 51421.116 & 139 & & & & \\
\hline & & 51421.254 & 126 & & & & \\
\hline 176232 & roAp & 51416.421 & 125 & SAAO: PM, KZ & $4400-7000$ & 37000 & \\
\hline \multirow[t]{2}{*}{ (10 Aql) } & & 51416.436 & 128 & & & & \\
\hline & & 51418.345 & 194 & & & & \\
\hline \multirow[t]{2}{*}{183806} & Ap & 51418.317 & 192 & SAAO: PM, KZ & $4400-7000$ & 37000 & \\
\hline & & 51418.330 & 206 & & & & \\
\hline \multirow[t]{2}{*}{212385} & Ap & 51416.466 & 159 & SAAO: PM, KZ & & & \\
\hline & & 51418.457 & 144 & & & & \\
\hline
\end{tabular}

made available from the archive of the Crimean Astrophysical Observatory (CrAO). The observational details and the reduction procedure for the ESO and SAAO spectra are briefly summarised in the following, for the CFHT spectra a description is given by Kochukhov et al. (2002a).

Excellent data of HD 29578, HD 60435 and HD 75445 were obtained with the Very Long Camera of the Coudé Echelle Spectrograph fibre-linked to the Cassegrain focus of the ESO 3.6-m telescope. The spectra of these three Ap stars were collected in February 2001 using the medium resolution CES image slicer and ESO CCD\#61. This instrumental setup allowed to record a $40 \AA$ spectral region centred at $\lambda 6136 \AA$ with a resolving power of $R \approx 123000$, according to the widths of emission lines in the Th-Ar comparison spectra.

Basic steps of spectroscopic reduction (bias subtraction, division by a normalized flat field, extraction of a $1 \mathrm{D}$ spectrum, continuum normalization and wavelength calibration) were performed with a set of IDL routines specially adapted for the optimum extraction of CES spectra. The typical signalto-noise ratio of our CES observations of Ap stars is about 200-250 per pixel.

In addition, we extracted CES observations of HD 29578, HD 75445, and HD 116114 from the ESO archive. These spectra were obtained with the instrumental configuration very similar to our February 2001 CES observing run (medium resolution image slicer, $R=122500$ ) and they cover the spectral region $\lambda \lambda 6127-6167 \AA$. The raw archival data were reprocessed with the same reduction software as was used in the analysis of our own CES observations resulting in extracted $1 \mathrm{D}$ spectra with a $S / N \approx 100-150$ per pixel.

Medium resolution spectra with $R \approx 37000$ were obtained with the echelle spectrograph GIRAFFE, mounted at the 1.9-m Radcliffe telescope of SAAO (South African Astronomical Observatory). They cover the wavelength region between 4400 and $7000 \AA$. We attempted to obtain a signal to noise ratio of at least 100 for each exposure and improved the quality of the spectra by co-adding several exposures taken during the same night.

The data reduction of the GIRAFFE spectra was based on IRAF. Bias subtraction, division by a normalized flat field, scattered light correction, wavelength calibration and extraction of the $1 \mathrm{D}$ spectra were done with standard routines. The normalization of the continuum was quite challenging in the regions where absorption lines with wide wings, especially $\mathrm{H} \alpha$, were present. In order to obtain satisfactory response functions for these parts of our spectra, a linear interpolation was applied 
between orders adjacent to those affected by a hydrogen line. Nevertheless, the resulting $\mathrm{H} \alpha$-line profiles were not used as the main temperature indicator for our spectrum analysis.

Besides the mentioned sources, we also used spectra obtained at the Crimean Astrophysical Observatory (CrAO) as described by Ryabchikova et al. (2001). Mainly Cr, Fe, Pr and $\mathrm{Nd}$ were investigated in the mentioned paper, therefore we decided to revise the CrAO spectra of HD 110066, HD 137909, and HD 188041 to obtain complete analyses including more elements.

\section{The global atmospheric parameters and the abundance analysis}

The initial set of atmospheric parameters $T_{\text {eff }}, \log g$ and $\log Z$ needed to start an abundance analysis were derived from Strömgren photometry using TEMPLOGG (Rogers 1995). Strömgren colour indices were taken from the catalogue of Hauck \& Mermilliod (1998) and from the Ph.D. Thesis of Martinez (1993).

A modified version of ATLAs9 (Kurucz 1993) was used to calculate scaled solar abundance model atmospheres. For some objects spectrophotometric observations were available, therefore $T_{\text {eff }}$ and $\log g$ could be derived from a fit of the theoretical energy distribution calculated with scaled solar abundance models to the observation (see Ryabchikova et al. 2000, 2001). For two stars, HD $137909=\beta \mathrm{CrB}$ and HD $137949=33 \mathrm{Lib}$, opacity distribution functions with individual abundances were calculated using a code developed by Piskunov \& Kupka (2001). The best fitting models were determined again from a comparison of the observed and synthetic flux distributions (Kupka et al. 2004). Lines contributing most to the line opacity for a given set of parameters were extracted from the Vienna Atomic Line Database (Piskunov et al. 1995; Ryabchikova et al. 1999; Kupka et al. 1999).

Usually, a difference between the effective temperatures obtained from Strömgren photometry and spectrophotometry does not exceed 200-300 K, and only for one star, HD 137949 , this difference amounts to $450 \mathrm{~K}$.

With the software package vwA, which is a semi-automatic procedure (Bruntt et al. 2002) in a combination with the WIDTH9 code (Kurucz 1993), a first quick-look analysis was produced. For stars with a $v_{\mathrm{e}} \sin i$ not larger than $20 \mathrm{~km} \mathrm{~s}^{-1}$, this method gives good starting values for a detailed analysis based on a comparison with synthetic spectra. An estimate of magnetic field effects on abundances was introduced at this stage.

The mean magnetic field modulus $\langle\mathrm{H}\rangle$ was determined from the Fe II Zeeman-doublet at $\lambda 6149.258 \AA$ by measuring the wavelength difference between the blue and red components, as described in Mathys et al. (1997). In cases of only partially resolved lines we tried to estimate the field value by comparing observations to synthetic line profiles calculated with the sYNTHMAG code (Piskunov 1999), where the magnetic field is taken into account in radiative transfer.

For the stars observed at SAAO a couple of other magnetically sensitive lines could be used to determine the mean magnetic field modulus. These lines were $\mathrm{Fe}_{\mathrm{I}} \lambda 6336.835 \AA$ $\left(g_{\text {eff }}=2.00\right)$ and Fe II $\lambda 6432.654 \AA\left(g_{\text {eff }}=1.83\right)$. Both lines produce a doublet-like Zeeman pattern. More details are given in a separate publication on the magnetic field discovery for HD 18610 (Stütz et al. 2003). A typical error of magnetic field measurements for ESO and CFHT spectra is about 50 to 70 Gauss, while it is 200 Gauss for SAAO spectra. The results of the magnetic field measurements are given in the last column of Table 1. Note that we co-added SAAO spectra and field values therefore refer to the mean HJD of the observations.

\section{Abundance determination}

Synthetic spectra taking into account the presence of a magnetic field were calculated for the entire observed spectral range for all stars observed at ESO. For the lower resolution spectra a magnetic spectrum synthesis was performed only for the small spectral parts which contain the lines chosen for an abundance analysis. Rotational velocities were estimated with synthetic spectra convolved with a set of $v_{\mathrm{e}} \sin i$ values. The typical error of $v_{\mathrm{e}} \sin i$ is $0.5 \mathrm{~km} \mathrm{~s}^{-1}$ for ESO spectra and $1-2 \mathrm{~km} \mathrm{~s}^{-1}$ for SAAO spectra.

For a few sharp-lined stars observed at SAAO (for example, HD 18610) the value derived for the rotational velocity is only an upper limit determined by the resolution of the spectrograph. For stars with $v_{\mathrm{e}} \sin i \geq 30 \mathrm{~km} \mathrm{~s}^{-1}$ the lack of visible magnetic splitting was compensated in our analysis by non-zero values for the microturbulent velocity.

The final abundances and atmospheric parameters for the program stars are summarized in Table 2. As a reference we do not give the usual solar abundances, but those of the normal late A-type star HD 32115, obtained with the same technique from spectra with compatible spectral resolution (Bikmaev et al. 2002). For a few rare-earth elements with no measurable lines in the spectra of normal stars we give the solar reference values from Grevesse \& Sauval (1998).

We have already mentioned that the atomic parameters for most spectral lines were extracted from VALD. In a few cases, however, oscillator strengths were corrected as is described by Bikmaev et al. (2002). One important aspect of the present abundance analysis was the $\mathrm{Pr}-\mathrm{Nd}$ anomaly and we therefore used the latest data on rare-earth elements published by Palmeri et al. (2000) for Ce II, Bord (2000) for Nd III, and Bord (private communication) for Pr III.

In the following subsections we comment on some of the stars which were (re)analyzed for the present investigation.

\subsection{Rapidly oscillating Ap (roAp) stars}

HD 12098: The pulsation with a period of $7.61 \mathrm{~min}$ was recently discovered for this northern Ap star by Martinez et al. (2000). The longitudinal magnetic field varies from -0.5 to $1.8 \mathrm{kG}$ with the most probable rotation period of 5.377 days (Lüftinger et al. 2003). Our estimate of the rotational velocity of the star is $v_{\mathrm{e}} \sin i=10 \pm 2 \mathrm{~km} \mathrm{~s}^{-1}$, yet it depends on the assumed surface magnetic field geometry and makes measurement of Zeeman line splittings difficult. A rough estimate of the surface magnetic field modulus indicates $\approx 6.5 \mathrm{kG}$. Abundances for HD 12098 are similar to those of $33 \mathrm{Lib}$ and HD 166473, in particular for $\mathrm{Ba}$ and REE elements 


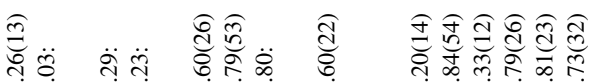

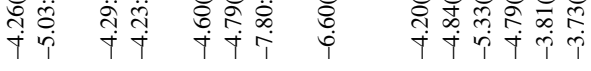

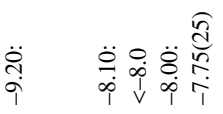

递

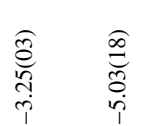

$\begin{array}{ll}\text { di } & \ddot{\sigma} \\ \dot{\alpha} & \dot{1}\end{array}$

के लू

ब⿳亠口冋.0.

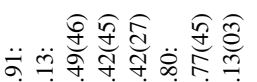

ह

望

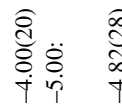

童总通

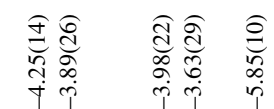

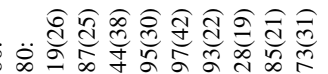

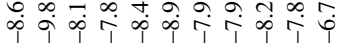

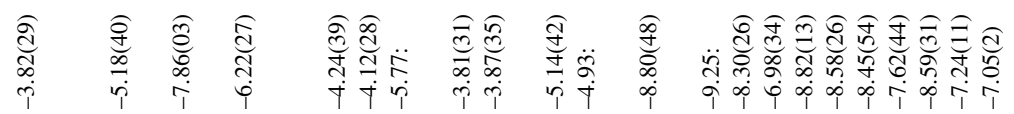

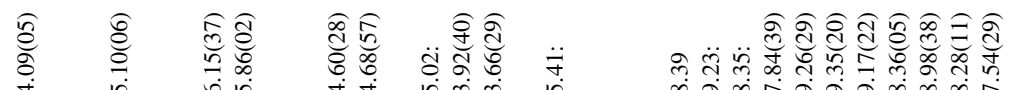

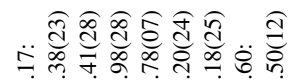

0
$\substack{\infty \\ \infty \\ \infty}$

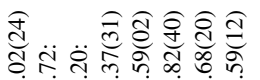

占

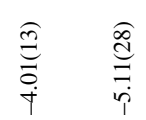

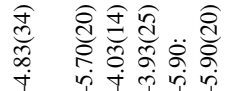

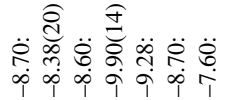

ธิ 


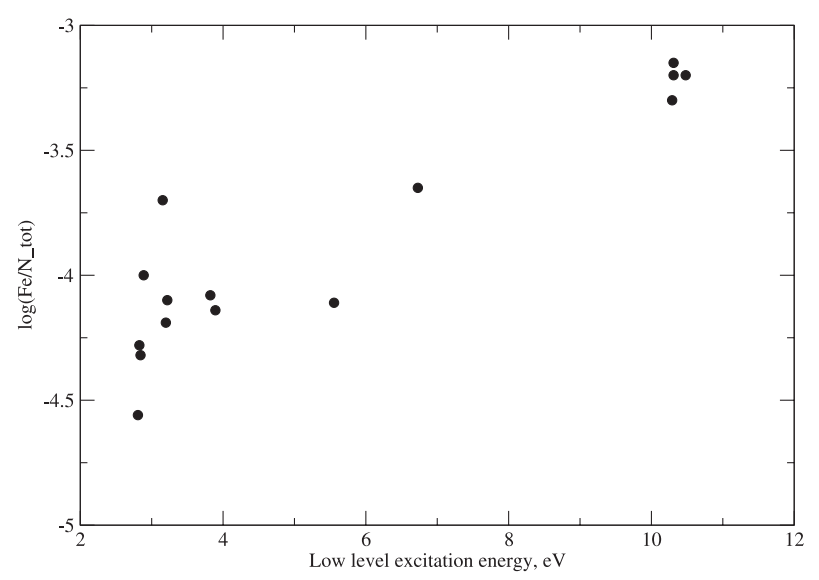

Fig. 1. Fe II abundance for 33 Lib (HD 137949) derived from individual lines with different low level excitation energies.

(Gelbmann et al. 2000 - Paper V). HD 166473 has the highest surface magnetic field among roAp stars and is followed by HD 12098, if our estimate of the mean surface magnetic field is correct.

HD 60435: For our analysis we had only the short spectral region from $6116-6156 \AA$ available. The Nd anomaly is present and the abundances of other elements are typical for cool roAp stars. The magnetic field is less than $5 \mathrm{kG}$ for this moderately rotating star, because we cannot see any splitting of the Fe II $\lambda 6149 \AA$ line. An upper limit of $2 \mathrm{kG}$ can be estimated from differential intensification of Fe II $\lambda 6147$ and $6149 \AA$ lines. For $\mathrm{Si}, \mathrm{Ca}, \mathrm{Fe}$ our results agree well with the abundances published by Shavrina et al. (2001), while there is a major disagreement for $\mathrm{Cr}$, $\mathrm{Sm}$ (our abundances are lower by $0.5-1.0 \mathrm{dex}$ ), $\mathrm{Ba}$ and $\mathrm{Nd}$ III (our abundances are higher by 0.5 dex). The small difference in the used effective temperatures cannot explain the discrepancies and we conclude that the most probable explanation is a spectrum variability of the star due to rotation and spots. The atmosphere of HD 60435 is very similar to the roAp star $\alpha$ Cir.

HD $137949=33 \mathrm{Lib}: 33 \mathrm{Lib}$ is one of the most peculiar roAp stars and its spectrum is severely blended due to a large overabundance of REE elements (in particular of $\mathrm{Nd}$ ), the low temperature and its large magnetic field. Despite the rather strong magnetic field we do not see a clear separation of the magnetically sensitive lines in SAAO spectra because of the limited spectral resolution. We therefore give only an upper limit for the mean magnetic field modulus.

33 Lib shows a remarkable abundance difference by more than 2 dex obtained from lines of the first and the second ions for $\mathrm{Pr}$ as well as $\mathrm{Nd}$, which indicates vertical stratification of these elements (Ryabchikova et al. 2003). As another stratification signature in the spectrum of this star we observe unusually strong high-excitation lines of $\mathrm{Cr}$ II and Fe II (Fig. 1) considering the given effective temperature. Abundances from these lines are not included in the mean values presented in Table 2. Obviously, for this star stratification effects should be studied in more detail.

HD $176232=10 \mathrm{Aql}$ : This star is our reference for a comparison of the present abundance determinations with

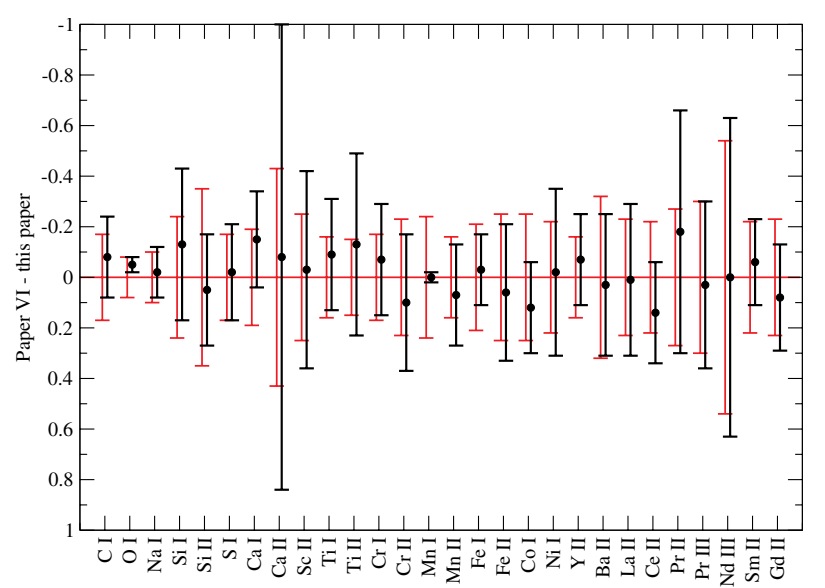

Chemical Element

Fig. 2. Comparison of abundances $\left(\log \left(N / N_{\text {tot }}\right)\right.$ derived for $10 \mathrm{Aql}$ (HD 176232) in this paper (dots) relative to Ryabchikova et al. (2000 Paper VI).

previous studies (see Papers I-VI). The analysis of $10 \mathrm{Aql}$ by Ryabchikova et al. (2000 - Paper VI) was based on observations of higher spectral resolution $(R=56000)$ obtained at McDonald Observatory, but with the same atmospheric parameters. Figure 2 compares present and previous abundance determinations and clearly indicates agreement within the quoted errors which justifies the combination of the present results with those from Papers I-VI.

\subsection{Non-oscillating Ap (noAp) stars}

HD 18610: We discovered a strong magnetic field for this star (Stütz et al. 2003). It is a typical cool Ap star and is similar to $\beta \mathrm{CrB}$ with a mild $\mathrm{Pr}-\mathrm{Nd}$ anomaly and high $\mathrm{Cr}$ abundance. HD 18610 also shows the same abundance increase for $\mathrm{Cr}$ and Fe lines with higher excitation energies.

Including also lines with $E_{\mathrm{i}}>7 \mathrm{eV}$ results in larger mean abundances and scatter $\left(\log \left(\mathrm{Cr} / N_{\text {tot }}\right)=-3.76 \pm 0.50\right.$, $\left.\log \left(\mathrm{Fe} / N_{\text {tot }}\right)=-3.13 \pm 0.60\right)$ which again is an evidence for chemical stratification.

HD 29578: Effective temperature, magnetic field strength and abundances are similar to $\beta \mathrm{CrB}$. The $\mathrm{Pr}-\mathrm{Nd}$ anomaly is higher than for $\beta \mathrm{CrB}$, but slightly lower than in roAp stars. It is comparable to that of HD 62140 (Ryabchikova et al. 2001), one of the roAp candidate stars. $T_{\text {eff }}$ qualifies this star as a (hotter) roAp candidate.

HD 29578 shows a remarkable magnetic field variability. Our two measurements of $\langle\mathrm{H}\rangle$ indicate a doubling of the mean magnetic field strength since the last published data (Mathys et al. 1997). Figure 3 (upper panel) displays measurements from Mathys et al. (circles) together with our measurements (diamonds). The magnetic period would be at least 12 years, if our observations were obtained close to the magnetic maximum, a presumption which remains to be confirmed.

HD 75445: The Pr-Nd anomaly in this star is very large and typical for roAp stars. Our three new magnetic field values are presented in the middle panel of Fig. 3. The mean magnetic field modulus seems to have slightly decreased during the last 7 years, indicating a very long period which is supported 


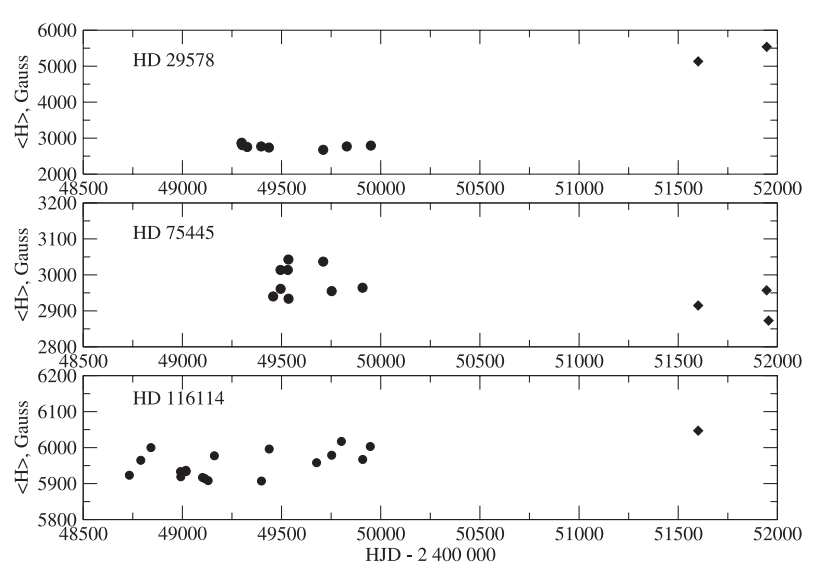

Fig. 3. Magnetic field variations for three program stars. Measurements from Mathys et al. (1997) are shown by circles, our measurements from ESO spectra are shown by diamonds.

also by extremely sharp spectral lines. In its long period, atmospheric parameters, magnetic field strength and abundance anomalies HD 75445 resembles the well known roAp star $\gamma$ Equ, thus we consider it an obvious candidate for the group of roAp stars. Up to now no pulsation signature exceeding an upper limit of one mmag could be detected (Martinez, private communication).

HD 110066: For the analysis of this star we used the same observations and atmospheric parameters as Ryabchikova et al. (2001), but determined abundances for additional elements. The higher effective temperature makes it more difficult to study the REE in the first ionization stage. Therefore the abundances derived from such lines are more uncertain. No $\mathrm{Pr}-\mathrm{Nd}$ anomaly is present. HD 110066 shows the highest $\mathrm{Cr}$ and $\mathrm{Fe}$ abundances among all program stars.

HD 116114: Both high (ESO) and lower (SAAO) resolution spectra were used for our analysis. The star has an abundance characteristic which is typical for cool Ap stars and no noticeable $\mathrm{Pr}-\mathrm{Nd}$ anomaly is present. In many respects the star is similar to $\beta \mathrm{CrB}$, including possible $\mathrm{Cr}$ and $\mathrm{Fe}$ stratification, however, it has a lower $\mathrm{Cr}$ abundance. Abundances obtained only from high excitation $\mathrm{Cr}_{\text {II }}(-4.74 \pm 0.25)$ and $\mathrm{Fe}$ II $(-3.64 \pm 0.13)$ lines differ significantly from those given in Table 2 .

Magnetic field measurements for this star are shown in Fig. 3 (bottom panel). Landstreet \& Mathys (2000) published a period of 27.6 days based on their magnetic field measurements. Because an epoch was not given for their Fig. 12, we cannot check if our value for the magnetic field modulus fits to their curve.

$H D 137909=\beta C r B$ : This star is one of the brightest and coolest Ap stars. It was checked several times photometrically for pulsation, but always found to be constant. A recent spectroscopic study by Kochukhov et al. (2002a), however, indicates a variable $\mathrm{Fe}_{\mathrm{I}}$ line at $\lambda 6165.36 \AA$ with a period of $11.5 \mathrm{~min}$ and an amplitude of $70 \mathrm{~m} \mathrm{~s}^{-1}$. Remarkably, no variations were found in REE lines which would be an opposite trend to roAp stars, where the largest RV amplitudes are found for REE lines, while Fe lines are constant within the error limits. This discovery basically was confirmed by
Hatzes \& Mkrtichian (2004) who measured an amplitude of $138 \mathrm{~m} \mathrm{~s}^{-1}$ for a spectral feature at $6272 \AA$ which the authors attribute to a $\mathrm{Ce}$ II line blended with a $\mathrm{Cr}$ II feature. Hatzes \& Mkrtichian question the pulsation evidence for $\beta \mathrm{CrB}$ from Kochukhov et al. (2002a) arguing with unspecified instrumental problems of the GECKo spectrograph used at CFHT. This suspicion, however, is difficult to understand, because of the well documented RV stability of the instrument of typically $15 \mathrm{~m} \mathrm{~s}^{-1}$ during a night and about twice as much over five nights (Walker et al. 2003). Furthermore, Kochukhov et al. simultaneously have observed a $\mathrm{Ca}$ I line which proved to be constant to within $20 \mathrm{~m} \mathrm{~s}^{-1}$ and which is only $1 \AA$ distant to the $\mathrm{RV}$-variable Fe line.

We know from $\gamma$ Equ that frequencies and mode amplitudes change from night to night (Martinez et al. 1996) due to a yet not understood excitation mechanism and due to interference of multiple modes. Hence, the different frequency and the larger amplitude measured for a Ce line by Hatzes \& Mkrtichian at a different rotation phase does not indicate a contradiction. The spectral resolution available for Hatzes \& Mkrtichian which is only half of that for the GECKo spectrograph may also be relevant. Certainly, significantly more high time resolved and top quality spectra are needed to investigate pulsations of $\beta \mathrm{CrB}$ which may turn out to be a key element in understanding roAp stars.

As it was mentioned by Kochukhov et al. (2002a) a detection of small amplitude $\mathrm{RV}$ variations in $\beta \mathrm{CrB}$ questions the existence of roAp stars as a separate group among cool Ap stars. One of the main distinctions between roAp and noAp stars seemed to be the REE anomaly which is much smaller or even absent for noAp stars. Previously, comparisons of the $\mathrm{Pr}$ and $\mathrm{Nd}$ abundances in roAp and noAp stars were based on 1 to 3 lines of each element in the first ionisation stage. Pr II and Nd II lines are very weak in the spectra of stars hotter than $8000 \mathrm{~K}$ even with a significant overabundance of these elements. In a spectrum of a star like $\beta \mathrm{CrB}$, which has a stratified atmosphere (Wade et al. 2001) with a large $\mathrm{Cr}$ and $\mathrm{Fe}$ overabundance in the hot atmospheric layers, plenty of rather strong high excitation lines of $\mathrm{Cr}$ II and $\mathrm{Fe}$ II contribute to blends. As a result, abundances of $\mathrm{Pr}$ and $\mathrm{Nd}$ derived from such blended lines may be overestimated and the REE anomaly underestimated. Therefore we carefully analyzed Pr II and $\mathrm{Nd}$ II lines in new $\beta \mathrm{CrB}$ spectra of very good quality obtained at CFHT using a more realistic model atmosphere based on individual abundances (Kupka et al. 2004). Indeed, the $\mathrm{Pr}-\mathrm{Nd}$ anomaly increases, but still remains much smaller than observed in roAp stars. The $\mathrm{Cr}$ abundance, however, is about an order of magnitude higher than in most roAp stars.

For the same reasons mentioned for $33 \mathrm{Lib}$, we did not include high excitation lines of $\mathrm{Cr}$ II and Fe II in the averaged values of most of the program stars given in Table 2. To be consistent we omitted them also in our $\beta \mathrm{CrB}$ results, however, when using all the measurable lines the corresponding abundances would be $\log \left(\mathrm{Cr} / N_{\text {tot }}\right)=-4.40$ and $\log \left(\mathrm{Fe} / N_{\text {tot }}\right)=-3.43$.

The mean magnetic field modulus derived from our spectra fits nicely a curve shown in Mathys et al. (1997, Fig. 32 therein). 
Table 3. Atmospheric parameters and abundances for magnetic Ap stars taken from the literature.

\begin{tabular}{crcccl}
\hline \hline \multicolumn{1}{c}{ Star } & \multicolumn{1}{c}{$T_{\text {eff }}$} & $\log g$ & $\log \left(\mathrm{Cr} / N_{\text {tot }}\right)$ & $\log \left(\mathrm{Fe} / N_{\text {tot }}\right)$ & Reference \\
\hline HD 43819 & $11300(100)$ & 3.20 & $-5.02(20)$ & $-3.66(20)$ & López-García \& Adelman (1994) \\
HD 79158 & $13300(300)$ & 4.00 & $-5.20(15)$ & $-3.50(15)$ & Wade et al. (2004) \\
HD 108662 & $10300(200)$ & 4.30 & $-3.11(35)$ & $-3.02(14)$ & Savanov et al. (1996) \\
HD 108945 & $8800(200)$ & 4.00 & $-4.80(20)$ & $-4.00(20)$ & Savanov et al. (1996) \\
HD 112413 & $11600(200)$ & 4.02 & $-5.30(20)$ & $-3.60(20)$ & Kochukhov et al. (2002b) \\
HD 133029 & $11200(100)$ & 3.84 & $-4.15(20)$ & $-3.28(20)$ & López-García \& Adelman (1999) \\
HD 153882 & $9250(200)$ & 3.85 & $-4.20(15)$ & $-3.13(15)$ & Ryabchikova et al. (1995) \\
HD 168733 & $14000(200)$ & 3.60 & $-5.20(25)$ & $-3.29(23)$ & this paper \\
HD 192913 & $10900(100)$ & 3.40 & $-4.92(50)$ & $-3.36(20)$ & López-García \& Adelman (1999) \\
HD 204411 & $8500(100)$ & 3.30 & $-5.04(20)$ & $-4.04(20)$ & Caliskan \& Adelman (1995) \\
HD 220825 & $9250(200)$ & 3.75 & $-4.30(20)$ & $-3.60(20)$ & Ryabchikova et al. (1996) \\
\hline
\end{tabular}

HD 183806: According to the temperature obtained from photometric calibrations, HD 183806 is the hottest star in our sample and has a rather large $v_{\mathrm{e}} \sin i$. Temperature and gravity determinations are consistent with the observed $\mathrm{H} \beta$ and $\mathrm{H} \alpha$ line profiles. The Hipparcos absolute visual magnitude $M_{\mathrm{V}}=-0.1 \mathrm{mag}$ (Gomez et al. 1998) gives together with $T_{\text {eff }} \approx 10000 \mathrm{~K}$ a stellar radius of about $3.7 R_{\odot}$. The rotation period $P=2.9213$ days was determined by Manfroid \& Mathys (1985) from photometry and infers an equatorial rotation velocity of $\approx 64 \mathrm{~km} \mathrm{~s}^{-1}$. With a measured $v_{\mathrm{e}} \sin i=28 \mathrm{~km} \mathrm{~s}^{-1}$ we can estimate the angle of inclination of the rotation axis to be $\approx 26^{\circ}$. $H D 188041=H R$ 7575: For this star abundance analyses based on photographic spectra in the blue region were published by Kato \& Sadakane (1999) and, for only a few elements, by Ryabchikova et al. (2001). The used effective temperature was the same for both studies, but gravity differed by 1 dex. Kato $\&$ Sadakane derived a $\log g=3.5$ mainly from hydrogen line profiles, while Ryabchikova et al. (2001) used a $\log g=4.5$ to better fit the spectrophotometry. Kato \& Sadakane (1999) noticed that their atmospheric parameters do not satisfy an ionization balance for iron, and in particular a few high excitation Fe II lines provided a very high iron abundance. Otherwise, our present analysis agrees very well with the results from Kato $\&$ Sadakane, and we again have to deal with a stratified atmosphere. This property may also result in different surface gravities derived from hydrogen lines and from a flux distribution, compared to an analysis based on a homogeneous atmosphere. A Pr-Nd anomaly is not observed in this star.

HD 212385: This star is one of the hottest in our sample of normal Ap stars, making again abundance determinations from lines of the first ionization stage rather uncertain. No Pr-Nd anomaly was detected. The rather large $v_{\mathrm{e}} \sin i=32 \mathrm{~km} \mathrm{~s}^{-1}$, determined with a scatter of up to $3 \mathrm{~km} \mathrm{~s}^{-1}$, prevented a measurement of the magnetic line splitting. Another magnetic field indicator could not be used either: the equivalent width ratio of the Fe II lines at $6147 \AA$ and $6149 \AA$. This ratio should be close to one in the non-magnetic case, otherwise the width of the $6147 \AA$ line is always larger. Unfortunately, the $6149 \AA$ line is blended with a yet unidentified spectral line at $6148.8 \AA$ which prevents a reliable equivalent width measurement. For our spectrum synthesis we used a microturbulent velocity of $4 \mathrm{~km} \mathrm{~s}^{-1}$ to compensate somehow for magnetic field effects.
Published magnetic Ap star abundances: For the purpose of better statistics in the investigation of the spectroscopic signature of roAp stars, in particular for the $\mathrm{Cr}$ and $\mathrm{Fe}$ abundances, we increased the temperature range of our sample by including data from the literature. For consistency reasons we limited the additional data to those determined in a similar way. If available, we also used the results from Doppler imaging and averaged over the entire stellar surface. A list of the supplement stars with $T_{\text {eff }}, \mathrm{Cr}$ and $\mathrm{Fe}$ abundances, and the references are given in Table 3. For HD 168733 the atmospheric parameters and equivalent widths of $\mathrm{Cr}$ and $\mathrm{Fe}$ lines were extracted from Muthsam \& Cowley (1984), but the abundances were recalculated with the same atomic parameters used by us for the present investigation.

\section{Spectroscopic signature of roAp stars}

A sample of 13 roAp and 12 non-pulsating Ap stars with temperatures ranging from $6400 \mathrm{~K}$ to $10000 \mathrm{~K}$ and with homogeneous abundance determinations is now available for a comparative study. This sample together with data published in Papers I-VI, Gelbmann (1998), Cowley et al. (2000), Ryabchikova et al. (2001) and Kochukhov (2003) obviously confirms that both groups do not differ significantly in rotation and magnetic field strength, but have a rather small overlap in $T_{\text {eff }}$. We tried to increase our sample of non-pulsating Ap stars and found the stars listed in Table 3 which corroborates the trend that roAp stars are cool and noAp stars are hot. The question can be even raised, if all cool chemically peculiar magnetic stars are pulsating.

Globally, we cannot find any obvious differences in the abundance pattern of Ap stars (Fig. 4) which do pulsate or not (or are candidates for pulsation). For the groups of peculiar stars presented in this figure the scatter bars are dominated by temperature effects (see for example our Fig. 5) and individual abundance differences (spots, e.g.), but not by actual measuring errors. No "error" bars are given for elements which were determined only for a single star of a sample. The error bars given for the abundances of a star with near solar type abundances, HD 32115, are taken from Bikmaev et al. (2002) and reflect the level of accuracy which can be achieved in the best cases. 


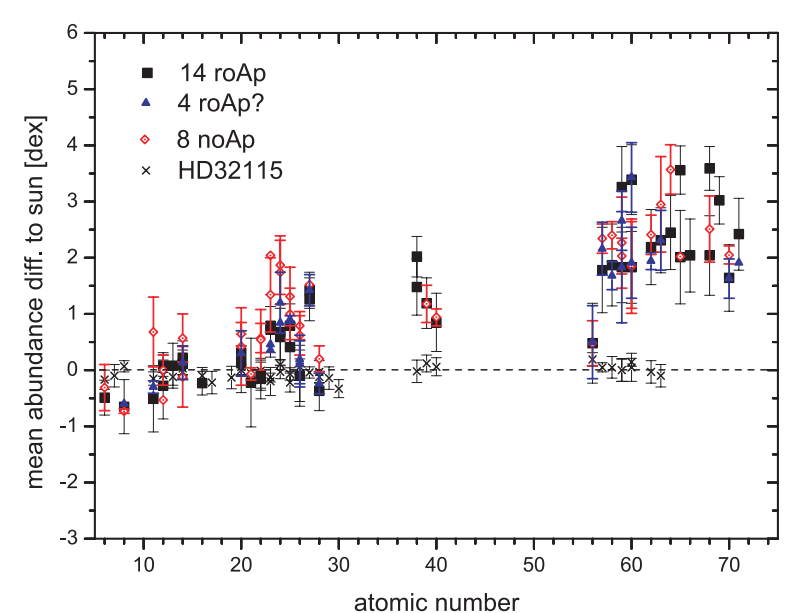

Fig. 4. Mean abundances for a sample of 13 roAp, 4 spectroscopic roAp star candidates, 10 non-pulsating Ap (noAp) stars. A well studied star (HD 32115, Bikmaev et al. 2002) with nearly solar abundances is presented for comparison. The error bars for HD 32115 are from the literature, whereas for the other groups they indicate the range (maximum - minimum) of values for the given sample.

A plot of the $\mathrm{Cr}$ and $\mathrm{Fe}$ abundances for roAp and noAp stars as a function of $T_{\text {eff }}$ shown in Fig. 5, results in an intriguing picture. Clearly, up to about $10500 \mathrm{~K}$ abundances increase with temperature, which immediately calls for another investigation: is there a similar trend for normal stars and/or is this trend artificially caused by incorrect model atmospheres and spectrum reduction techniques? For this purpose we compiled $\mathrm{Cr}$ and $\mathrm{Fe}$ abundances for normal, superficially-normal, Am and $\delta$ Scuti-type stars with low rotational velocities $\left(v_{\mathrm{e}} \sin i<50 \mathrm{~km} \mathrm{~s}^{-1}\right.$ ) from papers by Varenne \& Monier (1999, Hyades A and F stars), Hill \& Landstreet (1993), Mittermayer \& Weiss (2003), and from numerous papers by Adelman and co-workers: Adelman (1991, 1994, 1996, 1998, 1999), Adelman \& Davis Philip (1992), Adelman et al. (1997, 2000, 2001), Caliskan \& Adelman (1997), Adelman \& Albayrak (1998), Pintado \& Adelman (2003), and Kocer et al. (2003).

Compatibility of our results with literature data obtained with similar techniques has been discussed, e.g., by Ryabchikova et al. (2001). Furthermore, Grant Hill and John Landstreet kindly provided us access to spectra of 5 narrowlined A-type stars and the Am star Sirius for a direct comparison of our techniques with the one published in Hill \& Landstreet (1993). We calculated abundances from equivalent widths, using the same model parameters and a representative subsample of $\mathrm{Fe}$ and $\mathrm{Cr}$ lines. Like ours, the original analyses were based on ATLAs model atmospheres, but in combination with another spectrum synthesis code. Figure 6 shows that within the usual errors we obtained the same results and no systematic differences could be found.

The delta Scuti star included in Fig. 5 was analyzed by the same techniques described in this paper.

Back to Fig. 5: it is remarkable that the maximum abundance for $\mathrm{Cr}$ and $\mathrm{Fe}$ approaches the same value of $\log \left(N / N_{\text {tot }}\right)=-3$, which means an overabundance of $\mathrm{Cr}$ relative to the sun by a factor of 1000 and of about 30 for Fe. The process responsible for the element enrichment seems to be more efficient for $\mathrm{Cr}$. Above $10500 \mathrm{~K}$ the abundances for both elements drop drastically (more so for $\mathrm{Cr}$ ) and settle at a saturation point of about 10 times the solar value.

The radiative diffusion theory (Michaud 1970) seems to explain these effects. An abundance distribution (abundance profile) for a few iron peak elements was calculated by Babel (1992) for the atmosphere of the cool Ap star 53 Cam taking radiative diffusion in the presence of a weak stellar wind into account. The profile can be approximated by a step function with a low abundance in the upper atmospheric layers and a high abundance deeper inside. In principle this kind of atmospheric abundance profile could explain Fig. 5, if size and optical depth of the abundance jump change with effective temperature. For example, most lines with low- and medium-excitation energies would be formed in a region with low element abundance, if the abundance step is shifted towards deeper atmospheric layers. In this case a standard abundance analysis, assuming a homogeneous atmosphere, may even result in an underabundance as is indeed observed for Fe in HD 101065 and $\gamma$ Equ (Ryabchikova et al. 2003). Stratification analyses are presently available only for few stars (53 Cam, Babel 1992; $\gamma$ Equ, Ryabchikova et al. 2002; $\beta$ CrB, Wade et al. 2001; HD 133029, Monin et al. in preparation; HD 101065, Ryabchikova 2003) and seem to indicate a depth of $-0.5<\log \tau_{5000}<-1.2$ for such an abundance step with a difference of up to 3 dex.

It is worth mentioning that a similar, but considerably less prominent overabundance of $\mathrm{Cr}$ and $\mathrm{Fe}$ may be detectable for Am, normal and superficially normal stars around $T_{\text {eff }}=9500 \mathrm{~K}$. As all these stars do not show a measurable magnetic field it can be speculated that such a field significantly amplifies the process responsible for the observed abundances.

The most significant spectroscopic difference between roAp and non-pulsating Ap (noAp) stars is the REE anomaly. We compiled for Table 4 all available data concerning temperatures and abundance differences of second and first $\mathrm{Pr}$ and $\mathrm{Nd}$ ions, $\Delta[\mathrm{Pr}]_{\mathrm{III}-\mathrm{II}}$ and $\Delta[\mathrm{Nd}]_{\mathrm{III}-\mathrm{II}}$, together with an error estimate, given in parenthesis. For example, we show that most temperatures are uncertain to $100-200 \mathrm{~K}$, though a few are larger. Minor improvements of the published values concern HD 217522 for which Pr III and Nd III abundances were derived using the same spectra and model atmosphere as in Gelbmann (1998). We also increased slightly the temperature of the noAp star HD 184471 given by Ryabchikova et al. (2001). Recently, Carrier et al. (2002) found this star to be a long-period spectroscopic binary and they obtained $T_{\text {eff }}=8114 \mathrm{~K}$ from Geneva colours. This temperature is higher by $600 \mathrm{~K}$ than the one derived from Strömgren photometry (Renson et al. 1991). The spectrum of the companion might be detectable in the $\mathrm{H} \alpha$ line (Romanyuk, private communication) and the weakness of REE lines makes the analysis rather difficult and less accurate than for the other stars of the sample discussed in Ryabchikova et al. (2001). We therefore used a mean temperature derived from Geneva and Strömgren photometry and increased the error estimates for temperature and REE abundances.

The data from Table 4 are plotted in Fig. 7 and show a sharp change of the $\mathrm{Pr}-\mathrm{Nd}$ anomaly around $T_{\text {eff }}=8000 \mathrm{~K}$. All roAp stars (except of Nd in HD 101065, Przybylski's star) 

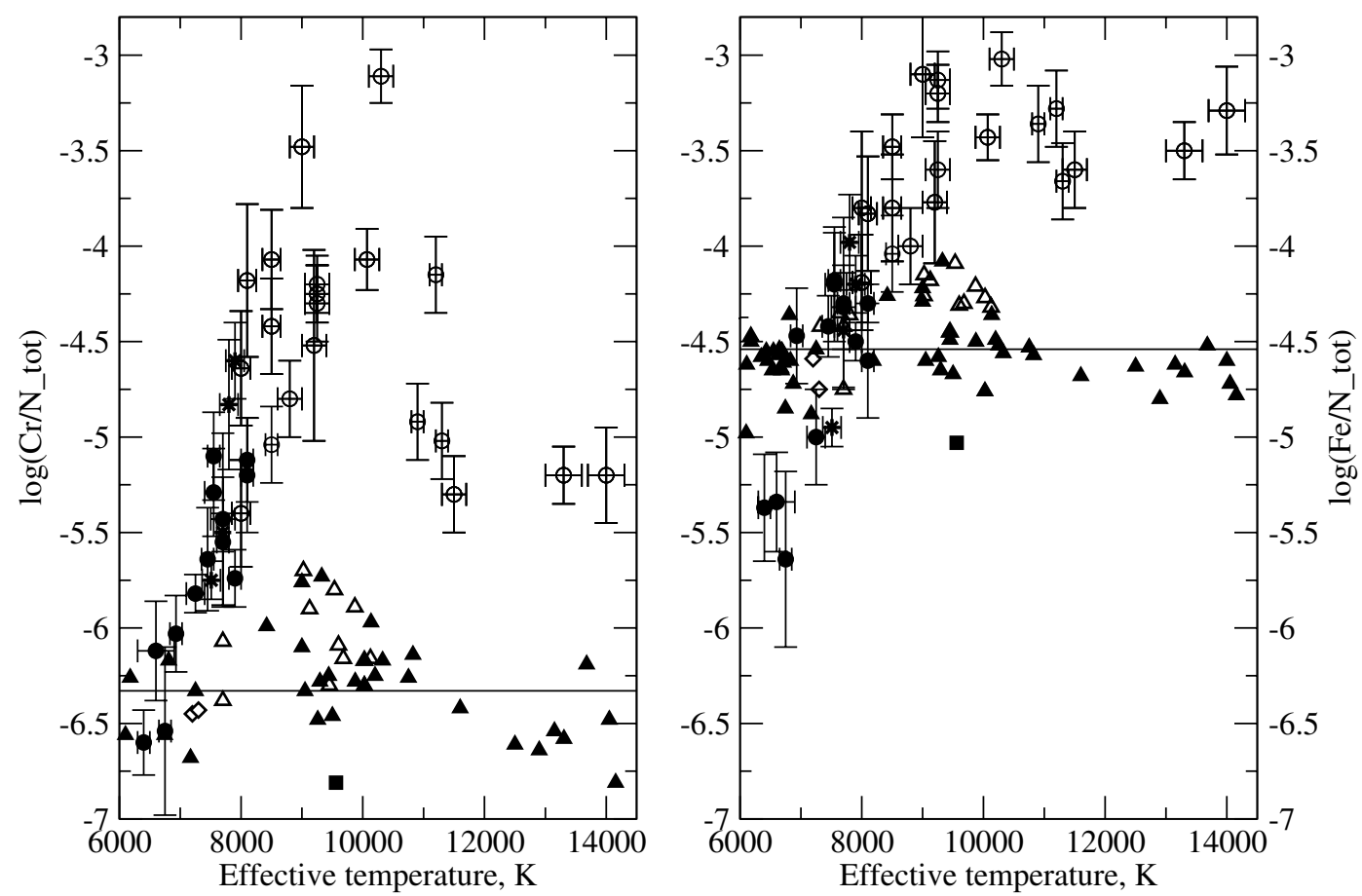

Fig. 5. $\mathrm{Cr}$ (left panel) and $\mathrm{Fe}$ (right panel) abundances versus effective temperature for 13 roAp stars (filled circles), 23 non-pulsating Ap stars (open circles) and 4 spectroscopic roAp candidates: HD 29578, HD 75445, HD 62140, and HD 115708 (asterisks). Also shown are abundances for normal and superficially normal stars (filled triangles), Am stars (open triangles), $\delta$ Scuti-type stars (open diamonds) and for Vega (filled square). Solar abundances are indicated by a horizontal line.
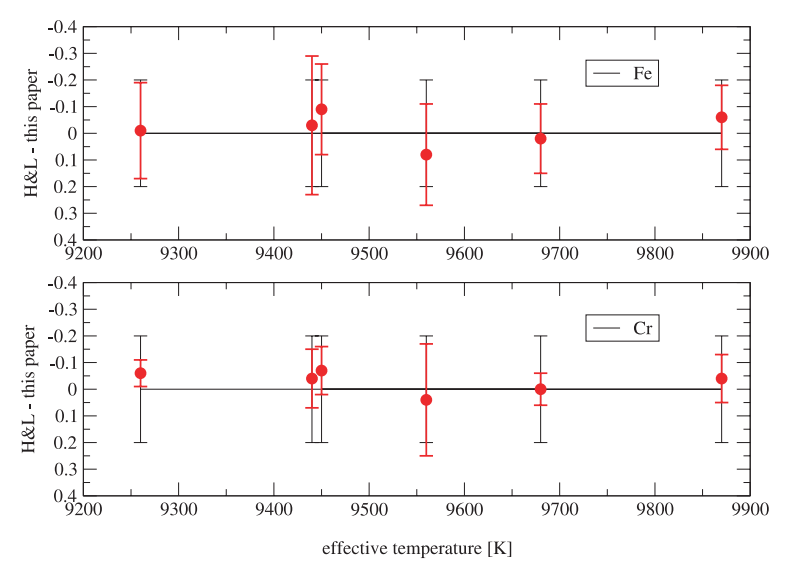

Fig. 6. Comparison of abundances $\left(\log \left(N / N_{\text {tot }}\right)\right.$ taken from Hill \& Landstreet $(1993-\mathrm{H} \& \mathrm{~L})$ relative to this paper.

have a $\operatorname{Pr}-\mathrm{Nd}$ anomaly of $\approx 1.5$ dex or even higher, while nonpulsating stars show marginal presence or even absence of the anomaly. We also see a very small temperature overlap for both groups of stars.

Obvious questions are:

- Is there a critical temperature below which (all?) magnetic Ap stars pulsate?

- Is the marginal temperature overlap of the group with and without REE anomaly real or mimicked by our procedures which basically are developed for normal homogeneous stellar atmospheres?

- What is the role of REE elements in general?
Table 4. Observed Pr and Nd anomaly.

\begin{tabular}{|c|c|c|c|}
\hline HD & $\bar{T} T_{\mathrm{eff}}$ & $\Delta[\mathrm{Pr}]_{\mathrm{III}-\mathrm{II}}$ & $\Delta[\overline{\mathrm{Nd}}]_{\mathrm{III}-\mathrm{II}}$ \\
\hline \multicolumn{4}{|c|}{ roAp stars } \\
\hline 12098 & $7800(150)$ & $1.50(30)$ & $1.80(30)$ \\
\hline 24712 & $7250(150)$ & $1.66(38)$ & $1.46(34)$ \\
\hline 42659 & $8100(100)$ & $1.34(38)$ & $1.08(32)$ \\
\hline 60435 & $8100(100)$ & & $1.50(40)$ \\
\hline 101065 & $6600(300)$ & $1.34(26)$ & $0.34(41)$ \\
\hline 122970 & 6930(100) & $1.41(37)$ & $1.20(37)$ \\
\hline 128898 & 7900(100) & $1.64(25)$ & $1.66(26)$ \\
\hline 137949 & $7550(150)$ & $2.19(38)$ & $2.21(57)$ \\
\hline 166473 & $7700(200)$ & $1.21(44)$ & $1.54(46)$ \\
\hline 176232 & $7550(100)$ & $0.75(58)$ & $2.55(69)$ \\
\hline 201601 & $7700(100)$ & $1.33(25)$ & $1.45(36)$ \\
\hline 203932 & $7450(100)$ & $1.29(44)$ & $1.92(37)$ \\
\hline 213637 & $6400(100)$ & $1.48(19)$ & $1.52(22)$ \\
\hline 217522 & $6750(100)$ & $1.42(28)$ & $1.52(51)$ \\
\hline \multicolumn{4}{|c|}{ roAp candidates } \\
\hline 29578 & $7800(150)$ & $0.62(40)$ & $1.10(40)$ \\
\hline 75445 & $7700(100)$ & $1.28(40)$ & $1.98(40)$ \\
\hline 62140 & $7900(150)$ & $0.80(39)$ & $1.29(25)$ \\
\hline 115708 & $7510(150)$ & $1.38(40)$ & $1.72(26)$ \\
\hline \multicolumn{4}{|c|}{ Non-pulsating Ap stars } \\
\hline 18610 & $8100(150)$ & $0.24(29)$ & $0.84(70)$ \\
\hline 110066 & $9000(200)$ & $-0.38(40)$ & $-0.36(45)$ \\
\hline 116114 & $8000(150)$ & $-0.22(31)$ & $0.14(45)$ \\
\hline 137909 & $8000(150)$ & $-0.09(35)$ & $0.81(23)$ \\
\hline 183806 & $10070(150)$ & $0.46(40)$ & $0.18(00)$ \\
\hline 184471 & 7800(400) & $0.30(50)$ & $0.03(50)$ \\
\hline 188041 & $8500(150)$ & $-0.51(48)$ & $0.04(47)$ \\
\hline 212385 & $9200(200)$ & $0.10(40)$ & $0.25(40)$ \\
\hline
\end{tabular}



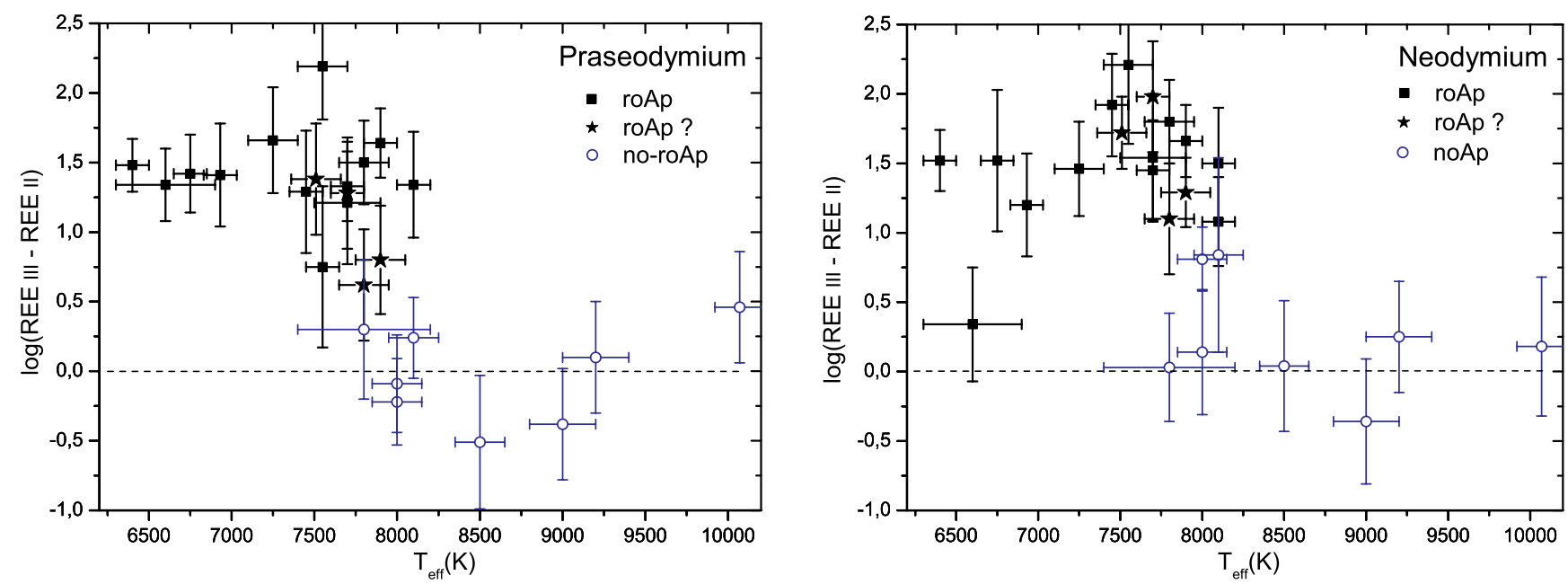

Fig. 7. Dependence of the $\operatorname{Pr}$ (left panel) and $\mathrm{Nd}$ (right panel) anomaly on temperature.

- How does stratification change the atmospheric structure and flux distribution?

\section{Conclusion}

The roAp and noAp stars are clearly different concerning the temperature domain they populate in the HR-diagram. The group of roAp stars is cooler than noAp stars with a fairly well defined border at $T_{\text {eff }}=8100 \mathrm{~K}$ and roAp stars are less metallic. As temperature increases, $\mathrm{Cr}$ and $\mathrm{Fe}$ abundances increase continuously to an upper level of approximately $\log \left(N / N_{\text {tot }}\right)=-3$ at about $T_{\text {eff }}=10500 \mathrm{~K}$ and decrease for hotter temperatures to a saturation level which is about 10 times of the solar value.

It is remarkable that a similar, but much less pronounced effect can be seen for "normal" and Am stars.

A definite spectroscopic signature of the roAp phenomenon seems to be an abundance differences of second and first ions of Pr and Nd of at least $1.5 \mathrm{dex}$ and up to $2.5 \mathrm{dex}$. The best confirmation of this indicator would be the photometric or spectroscopic discovery of pulsation in roAp candidates, e.g. from our Table 4. However, a more general picture of pulsation of CP2 stars discovered by Kochukhov et al. (2002a) may be indicated by the recent confirmation of pulsation in $\beta \mathrm{CrB}$ by Hatzes \& Mkrtichian (2004). All magnetic CP2 stars up to a transition temperature of about $8100 \mathrm{~K}$ may dispose of an excitation mechanism of yet unknown nature probably linked to diffusion processes. The REE anomaly and temperature dependence of $\mathrm{Cr}$ and $\mathrm{Fe}$ seems to be caused by radiative diffusion in the presence of a magnetic field (and stellar wind). If correct, we can quantify with Figs. 5 and 7 this process as a function of $T_{\text {eff }}$ and the magnetic field.

Acknowledgements. Grant Hill and John Landstreet kindly provided us access to their spectra for a direct comparison with our abundance determinations and the referee, Chuck Cowley, helped to improve the quality of our manuscript. This work was supported by the Fonds zur Förderung der wissenschaftlichen Forschung (project $P$-14984), by the Jubiläumsfonds der Österreichischen Nationalbank (project No. 7650), by the Lise Meitner fellowship to OK (project No. M757-N02), and by the Swedish Naturvetenskapliga
Forskningsrådet. T.R. thanks RFBR (grant 03-02-16342), Russian Federal program "Astronomy" (part 1102) and Leading Scientific School grant 162.2003.02 for partial financial support. We gratefully acknowledge the use of VALD and SIMBAD databases.

\section{References}

Adelman, S. J. 1991, MNRAS, 252, 116

Adelman, S. J. 1994, MNRAS, 271, 355

Adelman, S. J. 1996, MNRAS, 280, 130

Adelman, S. J. 1998, MNRAS, 296, 856

Adelman, S. J. 1999, MNRAS, 310, 146

Adelman, S. J., \& Albayrak, B. 1998, MNRAS, 300, 359

Adelman, S. J., \& Davis Philip, A. G. 1992, PASP, 104, 316

Adelman, S. J., Caliskan, H., Kocer, D., \& Bolcal, C. 1997, MNRAS, 288,470

Adelman, S. J., Caliskan, H., Kocer, D., Cay, I. H., \& Gokmen Tektunali, H. 2000, MNRAS, 316, 514

Adelman, S. J., Caliskan, H., Kocer, D., et al. 2001, A\&A, 371, 1078

Babel, J. 1992, A\&A, 258, 449

Bikmaev, I. F., Ryabchikova, T. A., Bruntt, H., et al. 2002, A\&A, 389, 537

Bord, D. 2000, A\&AS, 144, 517

Bruntt, H., Catala, C., Garrido, R., et al. 2002, A\&A, 389, 345

Caliskan, H., \& Adelman, S. J. 1995, in Astrophysical Applications of Powerful New Databases, ed. S. J. Adelman, \& W. L. Wiese, ASP Conf. Ser., 78, 443

Caliskan, H., \& Adelman, S. J. 1997, MNRAS, 288, 501

Carrier, F., North, P., Udry, S., \& Babel, J. 2002, 394, 151

Cowley, C. R., Ryabchikova, T., Kupka, F., et al. 2000, MNRAS, 317, 299

Gelbmann, M. 1998, Contrib. Astron. Obs. Scalnaté Pleso, 27, 280

Gelbmann, M., Kupka, F., Weiss, W. W., \& Mathys, G. 1997, A\&A, 319, 630 (Paper II)

Gelbmann, M., Ryabchikova, T. A., Weiss, W. W., et al. 2000, A\&A, 356, 200 (Paper V)

Gomez, A. E., Luri, X., Grenier, S., et al. 1998, A\&A, 336, 953

Grevesse, N., \& Sauval, A. J. 1998, Sp. Sci. Rev., 85, 161

Hatzes, A., \& Mkrtichian, D. 2004, MNRAS, in press

Hauck, B., \& Mermilliod, M. 1998, A\&AS, 129, 431

Hill, G. M., \& Landstreet, J. D. 1993, A\&A, 276, 142

Kato, K., \& Sadakane, K. 1999, PASJ, 51, 23 
Kocer, D., Adelman, S. J., Caliskan, H., Gulliver, A. F., \& Gokmen Tektunali, H. 2003, A\&A, 406, 975

Kochukhov, O., Landstreet, J. D., Ryabchikova, T., Weiss, W. W., \& Kupka, F. 2002a, MNRAS, 337, L1

Kochukhov, O., Piskunov, N., Ilyin, I., Ilyina, S., \& Tuominen, I. 2002b, A\&A, 389, 420

Kochukhov, O. 2003, A\&A, 404, 669

Kupka, F., Ryabchikova, T. A., Weiss, W. W., et al. 1996, A\&A, 308, 885 (Paper I)

Kupka, F., Piskunov, N., Ryabchikova, T. A., Stempels, H. C., \& Weiss, W. W. 1999, A\&AS, 138, 119

Kupka, F., Paunzen, E., \& Maitzen, H. M. 2004, A\&A, in press

Kurtz, D. W. 1978, IBVS, No. 1436, 1

Kurucz, R. L. 1993, CDROM13, SAO, Cambridge

Landstreet, J. D., \& Mathys, G. 2000, A\&A, 359, 213

López-García, Z., \& Adelman, S. J. 1994, A\&AS, 107, 353

López-García, Z., \& Adelman, S. J. 1999, A\&AS, 137, 227

Lüftinger, T., Ryabchikova, T. A., Weiss, W. W., et al. 2003, in International Conference on Magnetic Fields in O, B and A Stars: Origin and Connection to Pulsation, Rotation and Mass Loss, ed. L. A. Balona, H. F. Henrichs, \& R. Medupe, ASP Conf. Ser., 305, 92

Manfroid, J., \& Mathys, G. 1985, A\&AS, 59, 429

Martinez, P. 1993, Ph.D. Thesis, University of Cape Town

Martinez, P., Girish, V., Joshi, S., et al. 2000, IBVS, No. 4853

Martinez, P., Weiss, W. W., Nelson, M. J., et al. 1996, MNRAS, 282, 243

Mathys, G., Hubrig, S., Landstreet, J. D., Lanz, T., \& Manfroid, J. 1997, A\&AS, 123, 353

Michaud, G. 1970, ApJ, 160, 641

Mittermayer, P., \& Weiss, W. W. 2003, A\&A, 407, 1097

Monin, D. N., Wade, G. A., \& Landstreet, J. D., in preparation

Muthsam, H., \& Cowley, C. R. 1984, A\&A, 130, 348

Palmeri, P., Quinet, P., Wyart, J.-F., \& Biémont, E. 2000, Phys. Scr., 61,323

Pintado, O. I., \& Adelman, S. J. 2003, A\&A, 406, 987

Piskunov, N. E. 1999, in 2nd Workshop on Solar Polarization, ed. J. Stenflo, \& K. N. Nagendra (Dordrecht: Kluwer Academic Publishers), 515

Piskunov, N. E., Kupka, F., Ryabchikova, T. A., Weiss, W. W., \& Jeffery, C. S. 1995, A\&AS, 112, 525

Piskunov, N., \& Kupka, F. 2001, ApJ, 547, 1040
Renson, P., Kobi, D., \& North, P. 1991, A\&A, 89, 61

Rogers, N. Y. 1995, Comm. Asteroseismology, No. 78

Ryabchikova, T., Kuschnig, R., Piskunov, N. E., \& Pavlova, V. 1995, in Stellar Surface Structure, Poster, IAU Symp., 176, ed. K. G. Strassmeier, 132

Ryabchikova, T. A., Pavlova, V. M., Davydova, E. S., \& Piskunov, N. E. 1996, Astr. Lett., 22, 822

Ryabchikova, T. A., Adelman, S. J., Weiss, W. W., \& Kuschnig, R. 1997a, A\&A, 322, 234 (Paper III)

Ryabchikova, T. A., Landstreet, J. D., Gelbmann, M. J., et al. 1997b, A\&A, 327, 1137 (Paper IV)

Ryabchikova, T. A., Piskunov, N., Stempels, H. C., Kupka, F., \& Weiss, W. W. 1999, Phys. Scr., T83, 162

Ryabchikova, T. A., Savanov, I. S., Hatzes, A. P., Weiss, W. W., \& Handler, G. 2000, A\&A, 357, 981 (Paper VI)

Ryabchikova, T. A., Savanov, I. S., Malanushenko, V. P., \& Kudryavtsev, D. O. 2001, Astron. Rep., 45, 382

Ryabchikova, T., Piskunov, N., Kochukhov, O., et al. 2002, A\&A, 384, 545

Ryabchikova, T., Wade, G., \& LeBlanc, F. 2003, in Modelling of Stellar Atmospheres, ed. N. E. Piskunov, W. W. Weiss, \& D. F. Gray (ASP), IAU Symp., 210, 301

Ryabchikova, T. 1993, in Int. Conf., Magnetic Fields in O, B and A Stars: Origin and Connection to Pulsation, Rotation and Mass Loss, ed. L. A. Balona, H. H. Henriches, \& R. Medupe, ASP Conf. Ser., 305, 181

Savanov, I. S., Ryabchikova, T. A., \& Davydova, E. S. 1996, Astr. Lett., 22,815

Shavrina, A. V., Polosukhina, N. S., Zverko, J., et al. 2001, A\&A, 372, 571

Stütz, Ch., Ryabchikova, T., \& Weiss, W. W. 2003, A\&A, 402, 729

Varenne, O., \& Monier, R. 1999, A\&A, 351, 247

Wade, G. A., Ryabchikova, T. A., Bagnulo, S., \& Piskunov, N. 2001, in Magnetic fields across the Hertzprung-Russel diagram, ed. G. Mathys, S. K. Solanki, \& T. Wickramasinghe, ASP Conf. Ser., 248,341

Wade, G. A., Smith, M., Bohlender, D. A., et al. 2004, A\&A, submitted

Walker, G. H., Bohlender, D. A., Yang St. 2003, PASP, 115, 700

Weiss, W. W., Ryabchikova, T. A., Kupka, F., et al. 2000, in Impact of Large-Scale Surveys on Pulsating Star Research, ed. L. Szabados, \& D. W. Kurtz, ASP Conf. Ser., 203, 487 